\title{
Performance of Correlation in Topic Modeling from Academic Social Network Dataset through Deductive Learning
}

\author{
P. Sasikala \\ Research Scholar, Vels Institute of Science, \\ Technology \& Advanced Studies, VISTAS, Pallavaram, Chennai \\ Dr. P. Mayilvahanan \\ Professor, Department of Computer Science, Vels Institute of Science, \\ Technology \& Advanced Studies, VISTAS, Pallavaram, Chennai
}

\begin{abstract}
Large collections of documents are readily available online and widely accessed by diverse communities. Topic models can extract surprisingly interpretable and useful structure without any explicit "understanding" of the language by computer. The objective of this work to implement the leading machine learning algorithms, to get the optimal model through the one of the leading metric Matthew Correlation Coefficient. This work shows that the accuracies of the NaiveBayesMultinomialText classifier produces $64.59 \%$ level of accuracy, IBK classifier is $\mathbf{9 9 . 6 5 \%}$ level of accuracy,AdaBoostM1 classifier is $\mathbf{9 9 . 3 6 \%}$ and ZeroR classifier is $64.60 \%$ and DecisionStump classifier is $72.22 \%$. The DecisionStump algorithm, Instance based classifier and AdaBoost Classifiers are correlated positively, but this proposed system recommends that AdaBoost Classifier and IBK classifers are strongly correlated with this model.
\end{abstract}

Keywords: Topic Modeling, Correlation, Binary Classification, and Multi Classification

\section{INTRODUCTION}

In this paper we present the correlated topic model (CTM), which explicitly models the correlation between the latent topics in the collection, and enables the construction of topic graphs and document browsers that allow a user to navigate the collection in a topic-guided manner. The correlated topic model (CTM) is a hierarchical model of document collections. The CTM models the words of each document from a mixture model. The mixture components are shared by all documents in the collection; the mixture proportions are document specific random variables. The CTM allows each document to exhibit multiple topics with different proportions. It can thus capture the heterogeneity in grouped data that exhibit multiple latent patterns. The present study was focused on understanding the author's collaboration among research community in AMiner dataset.

The rest of this paper is organized as follows: Section 2 represents the materials and methods; Section 3 presents our results and discussions; then conclusion presents in Section 4.

\section{MATERIALS AND METHODS}

In this section presents the materials and methods of this research work. Two components were considered in this section. First one for extracting the themes in the article's abstract by topic modeling and the second one for classifying by using weka 3.8.3 version those identified topics into the domain subject area. The dataset collected was named as topic_paper_author in the academic social network data from https://aminer.org/topic_paper_author was shown in Table 1.

Table 1 Description of Topic_Paper_Author Dataset

\begin{tabular}{|c|c|c|}
\hline S.No. & Attribute & Data type \\
\hline 1 & Conference Name & String \\
\hline 2 & Title & String \\
\hline 3 & Year & Integer \\
\hline 4 & Abstract & String \\
\hline 5 & Authors & String \\
\hline
\end{tabular}

The dataset was collected for the purpose of cross domain recommendation. The attributes contain the following segmentation of subject areas.

$>$ Data Mining

$>$ Medical Informatics 


\section{$>$ Theory \\ $>$ Visualization \\ $>$ Database}

The first component in this experiment was realized by using the tool MeSH (Medical Subject Headings) (Topic Extraction Tool), which is recommended for extracting the topics for the abstracts available as a column/attribute in the dataset discussed earlier. The topics were divided into several categories, such as Algorithm, Data mining, Database, Artificial Intelligence, Clinical, Medical Imaging, Image Processing, Biomedical Informatics, Image Processing, and Telemedicine, which happens to be a little exercise around the topic modeling. These topic play the role of multiclass in this dataset.

Table 2: Class Distribution

\begin{tabular}{|c|c|c|c|}
\hline S. No. & Baseclass & Multiclass & No. of records \\
\hline 1 & \multirow[b]{5}{*}{ Computational } & Algorithm & 3999 \\
\hline 2 & & Artificial Intelligence & 630 \\
\hline 3 & & Data Mining & 2496 \\
\hline 4 & & Database & 4635 \\
\hline 5 & & Programming & 110 \\
\hline 6 & \multirow[b]{5}{*}{ Medicine } & Biomedical informatics & 961 \\
\hline 7 & & Clinical & 224 \\
\hline 8 & & Image Processing & 4064 \\
\hline 9 & & Medical Imaging & 1159 \\
\hline 10 & & Telemedicine & 97 \\
\hline
\end{tabular}

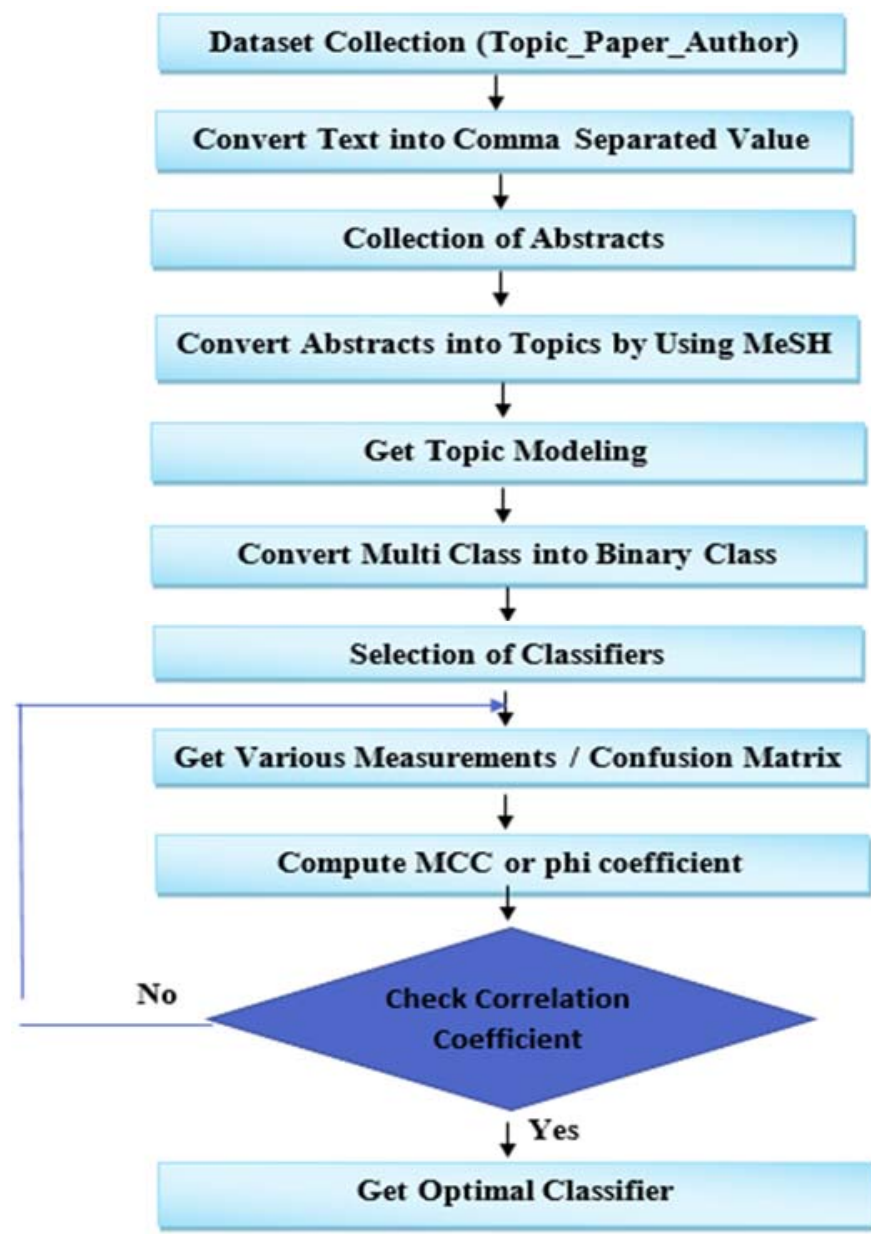

Figure 1: Architecture of Proposed System 
The second component in this experiment was realized by applying several classifiers for bringing out the better classification accuracy for categorizing the subject catalog for the given dataset, namely "Topic Paper Author" dataset. It contains 18,375 instances and 5 attributes.

\section{Methods:}

The Matthews correlation coefficient (MCC) or phi coefficient is used in machine learning as a measure of the quality of binary (two-class) classifications. The proportion of correct predictions (also termed accuracy), are not useful when the two classes are of very different sizes. For example, assigning every object to the larger set achieves a high proportion of correct predictions, but is not generally a useful classification.

$$
\mathrm{MCC}=\frac{T P \times T N-F P \times F N}{\sqrt{(T P+F P)(T P+F N)(T N+F P)(T N+F N)}}
$$

The above equation,

TP $=$ True Positive,

$\mathrm{TN}=$ True Negative,

$\mathrm{FP}=$ False Positive and FN=False Negative.

Confusion Matrix

\begin{tabular}{|l|l|l|}
\hline & Correct $\left(\mathrm{A}_{\mathrm{i}}\right)$ & Wrong $\left(\mathrm{B}_{\mathrm{j}}\right)$ \\
\hline Correct $\left(\mathrm{A}_{\mathrm{i}}\right)$ & $\mathrm{AB}_{11}[$ True Positive $]$ & $\mathrm{AB}_{10}[$ FalsePostive $]$ \\
\hline Wrong $\left(\mathrm{B}_{\mathrm{j}}\right)$ & $\mathrm{AB}_{01}[$ False Negative $]$ & $\mathrm{AB}_{00}[$ TrueNegative $]$ \\
\hline
\end{tabular}

The below machine learning classifications compute for finding the optimal classification algorithm for this research work.

$$
\begin{aligned}
& >\text { Bayes } \\
& >\text { Lazy } \\
& >\text { Meta } \\
& >\text { Rules } \\
& >\text { Trees }
\end{aligned}
$$

\section{RESULTS AND ANALYSIS}

In this section discusses results and analysis of this research work. This proposed work focuses on the computation and model optimization based on the one of the leading metrics namely Matthews correlation coefficient from the various machine learning algorithms like NaiveBayesMultinolialText classifer. It is under Bayes classifier. Then Instance based classifier or Lazy classifier. It belongs to Lazy category. Then AdaBoostM1 classifier belongs to Ensemble classifier, ZeroR classifier from Rules Based classifier and finally the DecisionStump classifier belongs to Trees classifier.

Table 3: Accuracy level for Various Classifiers

\begin{tabular}{|c|c|c|c|}
\hline S.No & Category of the Classifier & Name of the Classifier & Accuracy \\
\hline 1 & Bayes & NaiveBayeMultinomialText & $64.59 \%$ \\
\hline 2 & Lazy & IBK(k=1) & $99.65 \%$ \\
\hline 3 & Meta & AdaBoostM1 & $99.36 \%$ \\
\hline 4 & Rules & ZeroR & $64.60 \%$ \\
\hline 5 & Trees & DecisionStump & $72.22 \%$ \\
\hline
\end{tabular}

The above table represents the NaiveBayesMultinomialText classifier produces $64.59 \%$ level of accuracy, IBK classifier is $99.65 \%$ level of accuracy,AdaBoostM1 classifier is $99.36 \%$ and ZeroR classifier is $64.60 \%$ and DecisionStump classifier is $72.22 \%$. 
Table 4: Classifiers with Confusion Matrix Representation

\begin{tabular}{|c|c|c|c|}
\hline S.No & Category of the Classifier & Name of the Classifier & Confusion Matrix \\
\hline 1 & Bayes & NaiveBayeMultinomialText & {$\left[\begin{array}{cc}11870 & 0 \\
6505 & 0\end{array}\right]$} \\
\hline 2 & Lazy & IBK $(\mathrm{k}=1)$ & {$\left[\begin{array}{cc}11860 & 10 \\
54 & 6451\end{array}\right]$} \\
\hline 3 & Meta & AdaBoostM1 & {$\left[\begin{array}{cc}11869 & 1 \\
300 & 6205\end{array}\right]$} \\
\hline 4 & Rules & ZeroR & {$\left[\begin{array}{cc}11870 & 0 \\
6505 & 0\end{array}\right]$} \\
\hline 5 & Trees & DecisionStump & {$\left[\begin{array}{cc}11870 & 0 \\
5106 & 1399\end{array}\right]$} \\
\hline
\end{tabular}

The above table represents that the $\{\mathrm{TP}, \mathrm{FP}, \mathrm{FN}, \mathrm{TN}\}$ for various algorithms. Namley, NaiveBayeMultinomialText classifier has $\{11870,0,6505,0\}, \operatorname{IBK}(\mathrm{K}=1)$ classifier has $\{11860,10,54,6451\}$, AdaBoostM1 classifier has $\{11869,1,300,6205\}$, ZeroR Classifer has $\{11870,0,6505,0\}$, and DecisionStump Classifier has $\{11870,0,5106,1399\}$.

Table 5: Distribution of Matthews Correlation Coefficient

\begin{tabular}{|c|c|c|c|}
\hline S.No & Category of the Classifier & Name of the Classifier & MCC(phi-coefficient ( $\boldsymbol{\varphi})$ ) \\
\hline 1 & Bayes & NaiveBayeMultinomialText & 0 \\
\hline 2 & Lazy & IBK(k=1) & 0.99 \\
\hline 3 & Meta & AdaBoostM1 & 0.96 \\
\hline 4 & Rules & ZeroR & 0 \\
\hline 5 & Trees & DecisionStump & 0.39 \\
\hline
\end{tabular}

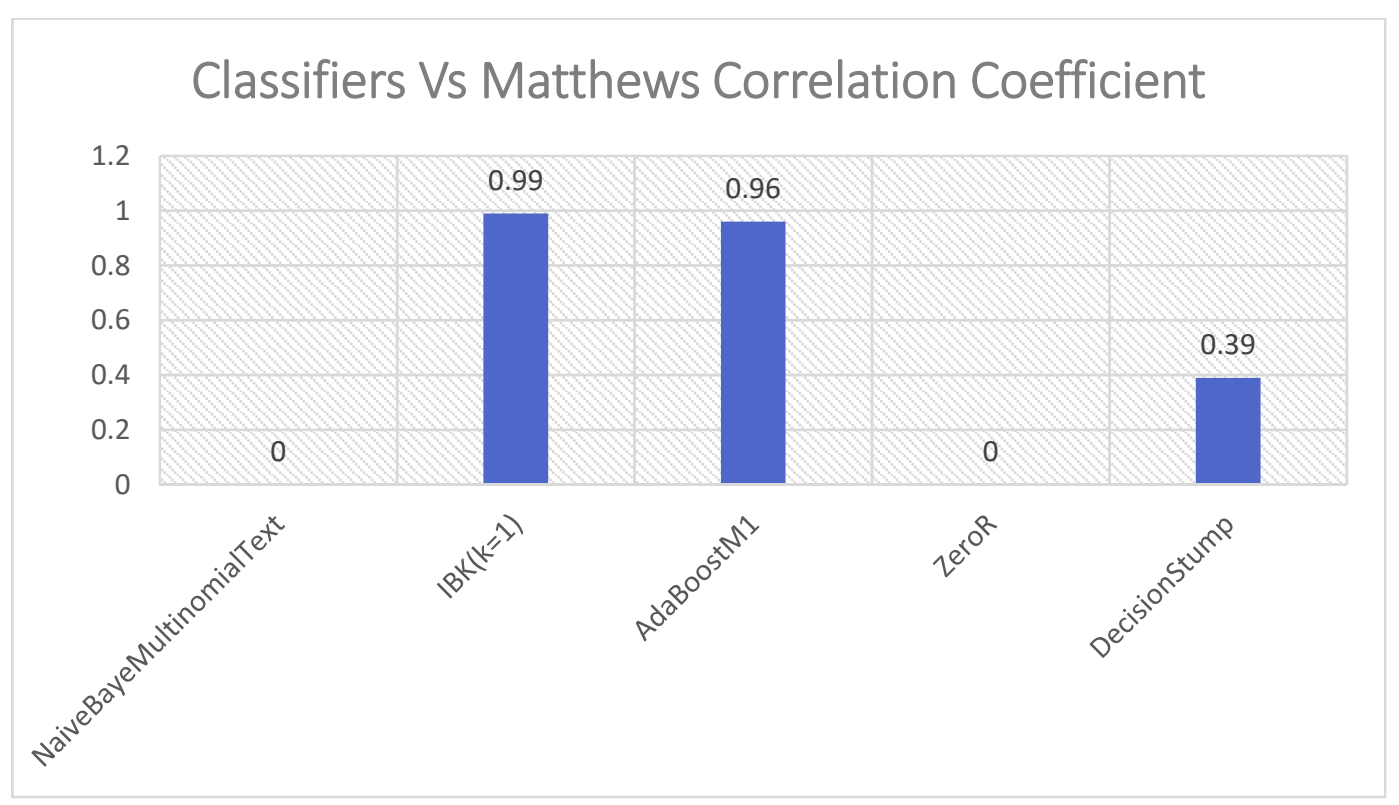

Figure 2 Graphical representations of various classifiers with their Correlation

The figure 2 clearly demonstrates that Mattheews Correlationn Coefficient values are various classifiers. Namely, the NaiveBayesMultinomialText classifier value is zero. The lazy classifier value is 0.99 , The AdaBoostM1 classifier value is 0.96, ZeroR classifier value is Zero and DecisionStump Classiffier value is 0.39.

So that it is representing the NaiveBayesMultinomialText belongs to Bayes Category classifier and ZeroR belongs to Rules category. These two classifiers are negatively correlated for this model. The DecisionStump is weakly correlated and Instance based classifier and AdaBoostM1 classifier are strongly correlated with positively. 


\section{CONCLUSION}

In this research work concludes that the based on the Matthews correlation coefficient metrics computed for confusion matrix of various leading machine learning algorithms, The DecisionStump algorithm, Instance based classifier and AdaBoostM1 Classifiers are correlated positively, but this proposed system recommends that AdaBoostM1 Classifier and IBK classifiers are strongly correlated with this model. And also Instance based classifier and AdaBoostM1 Classifiers are having above 99\% accuracy level.

\section{REFERENCES}

[1] Jie Tang, Sen Wu, Jimeng Sun, and Hang Su. Cross-domain Collaboration Recommendation. In Proceedings of the Eighteenth ACM SIGKDD International Conference on Knowledge Discovery and Data Mining (KDD'2012).

[2] Ayyappan.G et al. Knowledge Structure Infusion for Classification in Supervised Learning in Data Mining, International Journal on Computer Science and Engineering (IJCSE), Vol. 10 No.5 Apr-May 2018, Page No: 115-119,e-ISSN : 0975-3397, p-ISSN : $2229-5631$.

[3] https://meshb.nlm.nih.gov/MeSHonDemand

[4] J. M. Hofman, A. Sharma, and D. J. Watts, "in Social Systems," vol. 488, no. February, pp. 486-488, 2017.

[5] Ayyappan.G et al. Heart Disease Data Set Classifications: Comparisons of Correlation Co Efficient by Applying Various Parameters in Gaussian Processes, Indian Journal of Computer Science and Engineering (IJCSE), Volume No.9 Issue No.5 Oct-Nov 2018, Page No: 130-134, e-ISSN: 0976-5166, p-ISSN: 2231-3850.

[6] https://arxiv.org/pdf/0708.3601.pdf

[7] Ayyappan.G et al. Identification of Leading Research Contributors with Novel Performance Metrics Using Academic Social Network, International Journal on Computer Science and Engineering (IJCSE), Vol. 9 No.8 September 2017, Page No: 580-584,e-ISSN : 09753397, p-ISSN : 2229-5631.

[8] https://towardsdatascience.com/the-best-classification-metric-youve-never-heard-of-the-matthews-correlation-coefficient3 bf50a2f3e9a

[9] Ayyappan.G et al. A study on SNA: Text Mining using Academic Social Networks, International journal of Engineering and Technology (IJET), Volume No.8, Issue No.6, December 2016-Jan 2017, Page No: 2787-2790. ISSN: 0975-4024.

[10] http://arnetminer.org/lab-datasets/crossdomain/

[11] Ayyappan.G et al. A Novel K-NN Classification Approach Using Topic Modelling in Aminer Dataset, Indian Journal of Computer Science and Engineering (IJCSE), Volume No.10 Issue No.2 Apr-May 2019, Page No: 40-44, e-ISSN: 0976-5166, p-ISSN: $2231-3850$.

[12] http://www.cs.waikato.ac.nz/ml/weka/

[13] Ayyappan.G et al. A Case Study on A Miner Dataset: Identifying leading research through various Models, Indian Journal of Computer Science and Engineering (IJCSE), Volume No.10 Issue No.3 Apr-May 2019, Page No: 45-53, e-ISSN: 0976-5166, p-ISSN: 2231-3850.

[14] https://en.wikipedia.org/wiki/Matthews correlation coefficient\#Multiclass case

[15] Ayyappan.G et al. Ensemble Classifications for Student Academics Performance Data Seta, Indian Journal of Computer Science and Engineering (IJCSE), Volume No.10 Issue No.1 Feb-Mar 2019, Page No: 31-34, e-ISSN: 0976-5166, p-ISSN: $2231-3850$. 